\title{
Bose-Einstein Condensation and Many-Body Localization of Rotational Excitations of Polar Molecules
}

\author{
M. P. Kwasigroch and N. R. Cooper \\ T.C.M. Group, Cavendish Laboratory, University of Cambridge, J. J. Thomson Avenue, Cambridge CB3 OHE, U.K.
}

\begin{abstract}
We study theoretically the collective dynamics of rotational excitations of polar molecules loaded into an optical lattice in two dimensions. These excitations behave as hard-core bosons with a relativistic energy dispersion arising from the dipolar coupling between molecules. This has interesting consequences for the collective many-body phases. The rotational excitations can form a Bose-Einstein condensate at non-zero temperature, manifesting itself as a divergent $T_{2}$ coherence time of the rotational transition even in the presence of inhomogeneous broadening. The dynamical evolution of a dense gas of rotational excitations shows regimes of non-ergodicity, characteristic of many-body localization and localization protected quantum order.
\end{abstract}

PACS numbers: 67.85.-5, 05.30.-d, 72.15.Rn

The ability to create and control gases of cold polar molecules has sparked great interest in the quantum manybody physics associated with long-range and anisotropic dipolar interactions [1-8]. These systems open up possibilities to create and to probe interesting many-body phases involving the positional and/or rotational degrees of freedom of the molecules [9]. For polar molecules loaded into deep optical lattices - with positional motion frozen out - the rotational excitations can be used to emulate interesting forms of quantum magnet [10-22]. Recent experiments [23] have shown evidence of the dipole-dipole interactions between molecules in different lattice sites, which appear as an additional source of decoherence of rotational excitations [23, 24].

In this paper, we study the many-body physics of the rotational excitations of polar molecules in a two-dimensional (2D) system. We show that in regimes of weak disorder, rather than causing decoherence [23, 24], the dipole-dipole interactions can in fact stabilize the coherence up to arbitrarily long times. This stability of coherence arises from the formation of a collective many-body phase with true long-range order. The essential physics arises from the power-law $\left(1 / r^{3}\right)$ form of dipolar interactions between molecules. This coupling causes the rotational excitations to behave as a gas of (hardcore) bosons with a relativistic dispersion $\delta \epsilon_{\mathbf{k}} \propto|\mathbf{k}|$ at small wavevector $\mathbf{k}$. In contrast to massive particles $\left(\delta \epsilon_{\mathbf{k}} \propto|\mathbf{k}|^{2}\right)$ this relativistic dispersion allows a Bose-Einstein Condensate (BEC) to exist in 2D at non-zero temperature. We show that the formation of this BEC phase leads to a resistance to decoherence of the rotational excitations formed by a microwave pulse, even in the presence of inhomogeneities that would broaden the rotational transition for uncoupled molecules. For very strong inhomogeneous broadening there is a phase transition into an uncondensed phase for which the initial coherence decays exponentially in time.

An important feature of the rotational excitations of polar molecules is that they are effectively isolated from any external heat bath. In the presence of disorder such systems are natural candidates to show many-body localization and nonergodic behaviour [25, 26] as originally envisaged by Anderson [27] in the context of disordered spin systems in solids.
Indeed, we shall show that in the presence of disorder the collective dynamics of the rotational levels does exhibit such non-ergodic behaviour, with a transition from BEC to an uncondensed phase which does not coincide with the equilibrium phase transition.

We investigate a system of polar molecules prepared initially in their ro-vibrational groundstate and loaded in a single quasi-2D layer subject to a deep square optical lattice. The molecules are assumed to be in a Mott phase with one molecule per lattice site, such that tunneling of molecules between lattice sites can be neglected. The relevant degrees of freedom are the rotational excitations of the molecules.

We consider the rotational excitations introduced by a resonant microwave (MW) pulse linearly polarized perpendicular to the $2 \mathrm{D}$ plane. This prepares the initial state

$$
|\Psi\rangle=\prod_{i=1}^{N}\left(\cos \frac{\theta}{2}|\downarrow\rangle_{i}+\sin \frac{\theta}{2} e^{i \phi}|\uparrow\rangle_{i}\right)
$$

where $|\downarrow\rangle_{i}$ is the rotational groundstate $\left(\ell=0, m_{\ell}=0\right)$ of the molecule at site $i$ and $|\uparrow\rangle_{i}$ is its $\ell=1, m_{\ell}=0$ rotational excited state (with $m_{\ell}$ the angular momentum projection normal to the 2D plane). The angles $\theta$ and $\phi$ are set by the MW pulse (amplitude and phase). These are uniform over the system $\left(\theta_{i}=\theta, \phi_{i}=\phi\right)$, giving a uniform density $\rho=\sin ^{2} \frac{\theta}{2}$ of rotationally excited states with complete phase coherence.

Dipole-dipole interactions allow resonant exchange of rotational excitations between pairs of molecules, as described by the quantum spin Hamiltonian [11, 23]

$$
\mathcal{H}_{\mathrm{dip}}=\frac{J_{0} a^{3}}{2} \sum_{i \neq j} \frac{1}{\left|\mathbf{R}_{i}-\mathbf{R}_{j}\right|^{3}}\left(S_{i}^{-} S_{j}^{+}+S_{j}^{-} S_{i}^{+}\right),
$$

where $S_{i}^{ \pm}$are the spin raising/lowering operators for the spin$1 / 2, a$ is the lattice constant, and $J_{0}=d^{2} / 4 \pi \epsilon_{0} a^{3}$ is the coupling strength for nearest neighbour sites in terms of the dipole matrix element $d$ between $|\uparrow\rangle$ and $|\downarrow\rangle$ (e.g. $J_{0} / h=52 \mathrm{~Hz}$ for $\mathrm{KRb}$ in a lattice with $a=532 \mathrm{~nm}[23]$ ). Note that, in 2D the resonant transfer preserves $m_{\ell}=0$, so states with $m_{\ell}= \pm 1$ remain unpopulated [28]. 
Since (1) is not an eigenstate of the Hamiltonian (2), the state will evolve in time. Our goal is to determine the longtime behaviour. The central question is: does the system retain long-range correlations in local phases $\phi_{i}$ ? If so, then the system will remain resistant to decoherence, leading to a divergent $T_{2}$ coherence time as measured in standard spin-echo experiments. We will show that dipole-dipole interactions can cause the system to be resistant to decoherence: both for the ideal system with only dipole interactions (2); and for systems with additional disorder [Eqn. [6], representing inhomogeneous broadening of the molecular rotational transition, provided this disorder is below a critical value.

$B E C$ in the disorder-free system - To discuss (de)coherence, it is convenient to recast the model in terms of hard-core bosons, with $|\downarrow\rangle$ an empty site and $|\uparrow\rangle$ an occupied site. Within this viewpoint, the prepared state (1) is a BEC. The question of survival of long-range coherence becomes: does the system remain a BEC at long times? We shall quantify the residual coherence by computing the condensate fraction.

In the boson picture, the Hamiltonian (2) is the hard-core limit of the Bose-Hubbard model. However, it has the interesting feature that the intersite hopping scales with distance as $1 / r^{3}$. This leads to a dispersion relation for the bosons $\epsilon_{\mathbf{k}}=J_{0} a^{3} \sum_{i} \frac{e^{i \mathbf{k} \cdot \mathbf{R}_{i}}}{\left|\mathbf{R}_{i}^{3}\right|}$ for which $\delta \epsilon_{\mathbf{k}} \equiv \epsilon_{\mathbf{k}}-\epsilon_{\mathbf{0}} \propto|\mathbf{k}|$ at small wavevectors. This relativistic dispersion has striking consequences for the thermodynamic phase diagram of the model. Specifically, for a 2D system of conventional massive particles with $\delta \epsilon_{\mathbf{k}} \propto|\mathbf{k}|^{2}$ there can be no BEC at non-zero temperature. (Thermal fluctuations diverge, and leave at most power-law order in the condensate phase [29, 30].) However, for relativistic particles, the density of states vanishes sufficiently rapidly close to $\mathbf{k}=0$ to allow BEC in $2 \mathrm{D}$ even at non-zero temperature. As we now show, this BEC phase can be achieved by MW excitation.

To discuss this, we first make the assumption that the time evolution of the initial state (1) is such that at long times the system thermalizes. (We shall discuss in detail below dynamical issues that can prevent thermalization.) Since time evolution under the Hamiltonian (2) conserves both energy and number of rotational excitations, the final (thermal) state has temperature and chemical potential for the bosons that are set by the initial state. Note that, because the energy scale $J_{0}$ in (2) is positive, the total energy of the prepared state is of the order of $J_{0}$ per lattice site and is also positive. That is, it is close to the maximum energy state of (2). Thus, the corresponding thermalized states will be at negative absolute temperature $T$. The condensed "groundstate" that we will expand around is this maximum energy state, which is reached in the limit $T \rightarrow 0$ from negative temperatures; we shall denote this the " $T=0^{-}$groundstate".

The thermodynamic properties for small negative temperatures can be well described by a spin-wave analysis. At a mean excitation density $\rho$, this analysis leads to the mean en- ergy

$$
\left\langle\mathcal{H}_{\text {dip }}\right\rangle_{T}=N J \rho(1-\rho)+\sum_{\mathbf{k} \neq \mathbf{0}}\left(E_{\mathbf{k}} n_{\mathbf{k}}(T)+F_{\mathbf{k}}\right)
$$

where $J=\epsilon_{\mathbf{k}=\mathbf{0}} \sim 9.0 J_{0}, n_{\mathbf{k}}(T)=\left[\exp \left(E_{\mathbf{k}} / k_{B} T\right)-\right.$ $1]^{-1}$ is the thermal boson occupation number, and $E_{\mathbf{k}}=$ $-\sqrt{\left(\epsilon_{\mathbf{k}}(1-2 \rho)^{2}-J\right)\left(\epsilon_{\mathbf{k}}-J\right)}$ and $F_{\mathbf{k}}=\frac{1}{2}\left[J+E_{\mathbf{k}}-\epsilon_{\mathbf{k}}(1-\right.$ $\left.\left.2 \rho+2 \rho^{2}\right)\right]$ are, respectively, the dispersion of elementary excitations and the zero-point fluctuations. Similarly, the condensate density is

$$
\rho_{0} \equiv \sum_{i, j} \frac{\left\langle S_{i}^{+} S_{j}^{-}\right\rangle}{N^{2}}=\rho(1-\rho)-\frac{1}{N} \sum_{\mathbf{k} \neq \mathbf{0}}\left(n_{\mathbf{k}}^{Q}+n_{\mathbf{k}}^{T}\right)(4)
$$

where $n_{\mathbf{k}}^{Q}=-\frac{F_{\mathbf{k}}}{E_{\mathbf{k}}}$ and $n_{\mathbf{k}}^{T}=\left(1-2 \frac{F_{\mathbf{k}}}{E_{\mathbf{k}}}\right) n_{\mathbf{k}}(T)$ are the numbers of particles depleted from the condensate into the $\mathrm{k}$ mode due to, respectively, quantum and thermal fluctuations computed within Bogoliubov theory. The state (1) has an initial condensate fraction $\rho_{0}=\rho_{0}^{\max } \equiv \rho(1-\rho)$ which is the maximum possible for hard-core bosons.

The energy of the prepared state (1) is a conserved quantity in the isolated system and is given by

$$
\left\langle\mathcal{H}_{\text {dip }}\right\rangle_{\text {prepared }}=N J \rho(1-\rho) .
$$

Within the mean-field approximation the prepared state would be the exact $T=0^{-}$groundstate. However, quantum fluctuations cause the exact $T=0^{-}$groundstate to differ (slightly) from this prepared state, such that the mean energy (5) corresponds to a (small) non-zero temperature. Under the assumption of thermalization, the prepared states will thermalize to the BEC phase provided that $\left\langle\mathcal{H}_{\text {dip }}\right\rangle_{\text {prepared }}$ is larger than the mean energy at the transition point $\left\langle\mathcal{H}_{\mathrm{dip}}\right\rangle_{T_{\mathrm{C}}}$ at which the condensate density vanishes, $\rho_{0}\left(T_{\mathrm{C}}\right)=0$.

Fig. 1 1 summarizes the results of the above analysis. We find that the zero-point corrections are small, so the energy of the prepared system is always very close to that of the $T=0^{-}$ groundstate. (The smallness of these corrections justifies our spin-wave approximation.) Hence, after thermalization, the system is in the ordered (BEC) phase for all densities $\rho$, so the microwave pulse creates a state with infinitely long-lived coherence.

The disordered system - So far we have considered the pure system without external sources of decoherence. In experiment it is natural to have sources of broadening of the rotational transition, from inhomogeneities of lightshift or random filling of the lattice. In current experiments [23], optical lattices are loaded with molecules at filling fractions much smaller than unity, circa 0.1 . This is the limit where disorder dominates and leads to decoherence of the prepared state (1).

Related disordered spin models have been extensively studied in 1D [24, 31, 32], where strong quantum fluctuations preclude any long-range order. Here, we study the effects of disorder in 2D, where true long-range order can survive. We use a mean-field approach, motivated by the fact that zero-point corrections are very small for the clean system, and further 


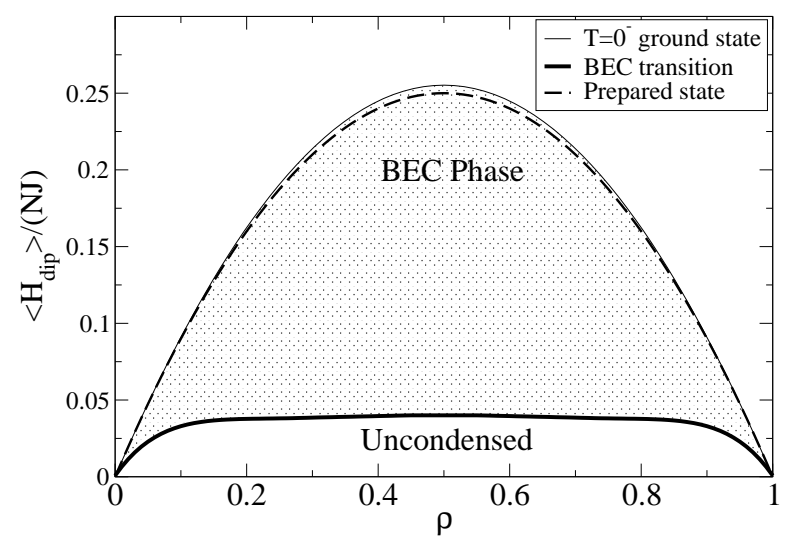

FIG. 1: Phase diagram of the disorder-free system. The prepared state is always close to the $T=0^{-}$groundstate because of small spin-wave corrections and hence thermalizes in the BEC phase for all $\rho$. The BEC transition line is the energy of the system corresponding to the transition temperature.

supported by beyond-mean-field treatments described below. The disorder enters through an onsite coupling

$$
\mathcal{H}_{\text {site }}=-\sum_{i}\left(\mu+\xi_{i}\right) S_{i}^{z}
$$

where $S_{i}^{z}= \pm 1 / 2$ for $|\uparrow\rangle /|\downarrow\rangle, \xi_{i}$ are Gaussian-distributed quenched random variables of standard deviation $W$ which sets the scale of inhomogeneous broadening. (For uncoupled spins, $J_{0}=0$, the initial state 11 would decohere with $T_{2} \sim$ $h / W$.) We have introduced a chemical potential $\mu$ to fix the density of rotational excitations $\rho$. Without loss of generality we take the mean-field to be along the $x$ direction

$$
B=\frac{2 J}{N} \sum_{i}\left\langle S_{i}^{x}\right\rangle .
$$

Within mean-field theory the total Hamiltonian $\mathcal{H}_{\text {site }}+\mathcal{H}_{\text {dip }}$ is replaced by

$$
\mathcal{H}_{\mathrm{MF}}=\sum_{i} \mathbf{S}_{i} \cdot \mathbf{B}_{i}-\frac{N B^{2}}{4 J}
$$

where $\mathbf{B}_{i}=\left(B, 0,-\mu-\xi_{i}\right)$. The spin on each site can now be treated independently. At equilibrium at temperature $T$ the mean-field satisfies the self-consistency equation

$$
1=J \int p(\xi) \frac{\tanh \left(\frac{1}{2 k_{B} T} \sqrt{B^{2}+(\mu+\xi)^{2}}\right)}{\sqrt{B^{2}+(\mu+\xi)^{2}}} d \xi
$$

where the summation over sites is equivalent to an ensemble average over $\xi$ in the thermodynamic limit, with $p(\xi)=$ $\left(2 \pi W^{2}\right)^{-1 / 2} \exp \left(-\xi^{2} / 2 W^{2}\right)$.

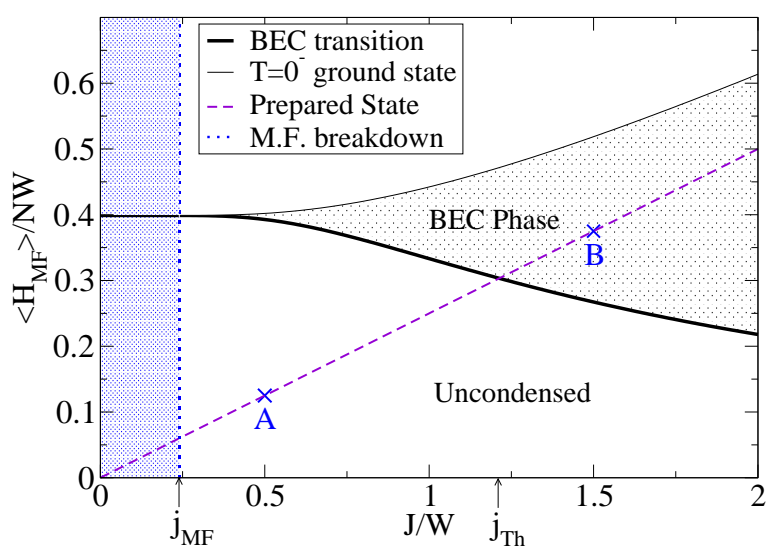

FIG. 2: Phase diagram of the disordered system for $\rho=\frac{1}{2}$. The BEC transition line is the energy of the system corresponding to the transition temperature. Isolated systems below this energy thermalize in the uncondensed phase. The dashed line is the energy of the prepared state with $\rho=\frac{1}{2}$ which thermalizes in the BEC phase for $J / W>j_{\mathrm{Th}}$. The mean-field description breaks down for $J / W<j_{\mathrm{MF}} \sim 0.24$. In Fig. 3 we compare the dynamics at points $\mathrm{A}$ and $\mathrm{B}$.

The solution of Eqn. (9) shows a phase transition between a BEC (with $B \neq 0$, giving condensate fraction $\rho_{0}=B^{2} / 4 J^{2}$ ) and uncondensed phases, at a critical temperature that depends on the disorder strength. Although mean-field theory shows no phase transition at zero temperature, in contrast to expectations of more accurate methods (e.g. [33, 34]), this weakness is unimportant for our purposes, since, as we now show, the transition of interest is in the regime $J / W>j_{\mathrm{MF}} \simeq$ 0.24 where mean-field theory is expected to capture the behaviour accurately [33].

Fig. 2 shows our results for $\rho=\frac{1}{2}$. The mean energy of the prepared state increases with $J / W$. For strong disorder, $J / W<j_{\mathrm{Th}} \simeq 1.2$, the mean energy of the prepared state is such that it is in a regime in which the thermalized state has no long-range order, so will completely decohere. For weak disorder, $J / W>j_{\mathrm{Th}} \simeq 1.2$, the mean energy of the prepared state places it in a regime in which the thermalized state forms a BEC and has long-range coherence. Hence, our results show that for sufficient dipolar coupling, $J / W \gtrsim 1.2$, the thermalized system will remain phase coherent despite the inhomogeneous broadening $(W \neq 0)$.

Dynamical Evolution of the Prepared State - Our results have been based on the assumption that the isolated system will reach thermal equilibrium at long times, with properties set only by the initial energy and excitation density $\rho$. We now turn to investigate the time evolution itself. We focus on the prepared state with density $\rho=1 / 2$, which is representative of other densities. Within the mean-field model, the dynamics of the expectation values of the individual spins obey

$$
\dot{\mathbf{S}}_{i}=\mathbf{S}_{i} \times \mathbf{B}_{i}
$$




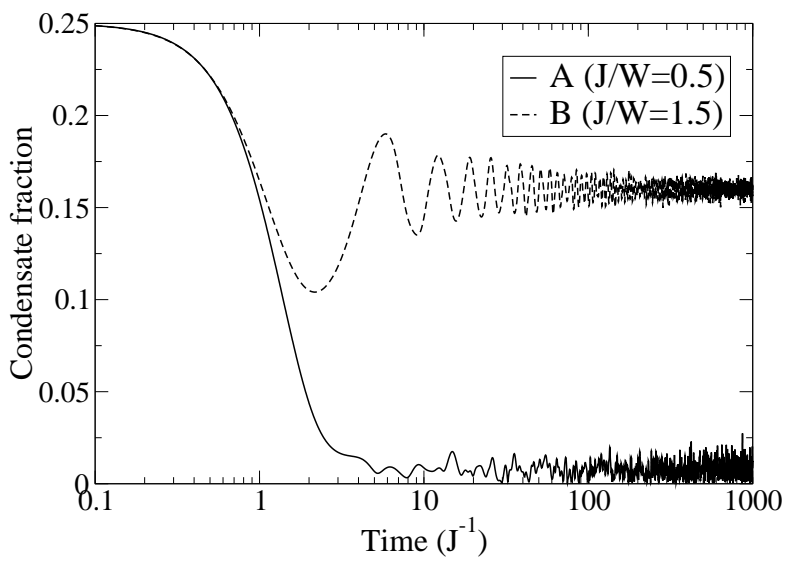

FIG. 3: Dynamical evolution of the condensate fraction, computed via $\rho_{0}=\frac{\left(B_{x}^{2}+B_{y}^{2}\right)}{4 J^{2}}$ from mean-field theory. For strong disorder the condensate fraction decays to zero at long times, so the initial state completely decoheres.

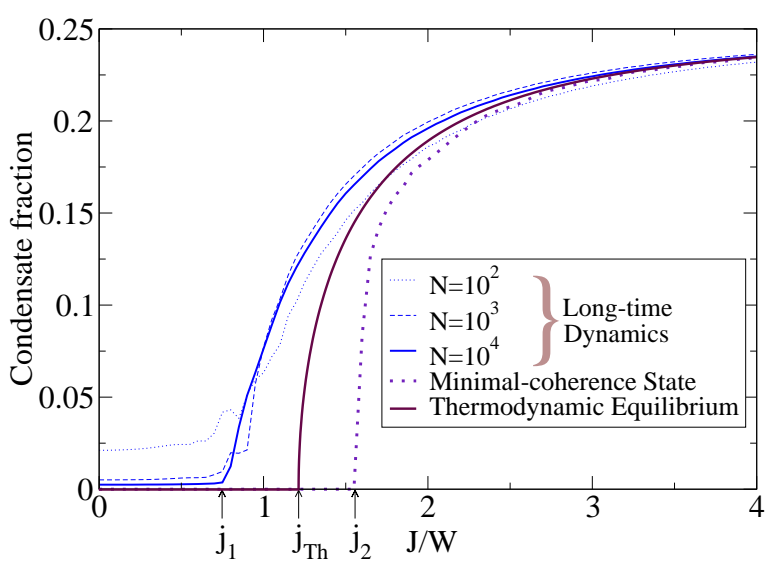

FIG. 4: Comparison of the condensate fraction for the thermalized state with that found from the long-time dynamics of mean-field theory (for increasing systems sizes $N$ ) for a system prepared with a coherent microwave pulse. The prepared state retains coherence in a regime $j_{1}<J / W<j_{\text {Th }}$ for which the thermalized state would be completely incoherent, showing non-ergodic dynamics in the disordered system. The dot-dashed line is the long-time behaviour for a system prepared in a state of minimal coherence, described in the text, with the same mean energy.

where $\mathbf{B}_{i}=\left(B_{x}, B_{y}, \xi_{i}\right)$ and $B_{x}, B_{y}$ are the mean-fields along the $x$ and $y$ directions respectively. We simulate this dynamics numerically for systems of up to $10^{5}$ spins, updating the mean- $B_{x}$ and $B_{y}$ fields at each time step.

Fig. 3 shows our results for two disorder strengths. Far inside the region where the thermalized state is uncondensed (A), the long time dynamics is to a state with vanishing con- densate fraction: i.e. the prepared state decoheres. Far inside the region where the thermalized state would be a BEC (B), the long-time dynamics shows a reduced, but non-zero residual condensate fraction: i.e. the system retains coherence to long times.

Thus we find that the dynamical evolution exhibits a transition between condensed (BEC) and uncondensed phases as a function of $J / W$, as we had also found based on the thermalization ansatz. However, the locations of these transitions differ. Fig. 4compares the long-time results of our dynamical simulation to the predictions based on thermodynamic equilibrium [35]. Thermodynamic and dynamical results converge for large and small $J / W$. However, they differ in the intermediate regime. For $j_{1}<J / W<j_{\text {Th }}$ the long-time dynamics of the system show long-range order, even though the thermalized system is disordered. This is a signature of many-body localization, and localization protected quantum order [25].

That the system does not reach thermal equilibrium for intermediate $J / W$ shows that the dynamics are non-ergodic, and sensitive to initial conditions. We have explored this further by simulating the evolution of a range of systems with different initial states of the same total energy: from the prepared state with maximal coherence (1); to a state of minimal coherence in which $S_{i}^{z}$ has the same sign as $\xi_{i}$ and we choose the maximum $\left|S_{i}^{z}\right|$ allowed by energy conservation. The dynamics of this state of minimal coherence also shows non-ergodic behaviour. In the intermediate range $j_{1}<J / W<j_{2}$ the system is locked in the initial state, be it coherent or incoherent, depending on the initial state (maximum or minimum coherence) and irrespective of the form of the thermalized state. Note that the mean-field theory we study is a classical nonlinear dynamical system. Similar non-ergodic behaviour, and lack of thermalization, has also been found in recent work by Tieleman et al. [26], who have shown that in the closely related mean-field theory of the Bose-Hubbard model, disorder breaks the connection between chaoticity and ergodicity even when classical localization cannot occur. We interpret the non-ergodic nature of the mean-field spin system as indicative of the many-body localization in the underlying quantum spin system.

In summary, we have shown that due to dipole-dipole interactions the rotational excitations of polar molecules in $2 \mathrm{D}$ optical lattices can form a BEC even at non-zero temperature. This collective many-body phase gives rise to longlived coherence of the rotational excitations, even in the presence of disorder that would otherwise cause inhomogeneous broadening. Our results show that experimental studies of the (de)coherence of the rotational excitations prepared by a microwave pulse, can be used to explore regimes of many-body localization, where non-ergodic behaviour allows long-range phase coherence even when the thermalized phase would be incoherent.

We gratefully acknowledge financial support from EPSRC, Grant No. EP/J017639/1. 
[1] J. Deiglmayr et al., Phys. Rev. Lett. 101, 133004 (2008).

[2] K.-K. Ni et al., Science 322, 231 (2008).

[3] S. Ospelkaus et al., Phys. Rev. Lett. 104, 030402 (2010).

[4] S. Ospelkaus et al., Science 327, 853 (2010).

[5] K.-K. Ni et al., Nature 464, 1324 (2010).

[6] K. Aikawa et al., Phys. Rev. Lett. 105, 203001 (2010).

[7] M. H. G. de Miranda et al., Nat. Phys. 7, 502 (2011).

[8] A. Chotia et al., Phys. Rev. Lett. 108, 080405 (2012).

[9] M. A. Baranov, M. Dalmonte, G. Pupillo, and P. Zoller, Chemical Reviews 112, 5012 (2012).

[10] A. Micheli, G. K. Brennen, and P. Zoller, Nat. Phys. 2, 341 (2006).

[11] R. Barnett, D. Petrov, M. Lukin, and E. Demler, Phys. Rev. Lett. 96, 190401 (2006).

[12] H. Büchler, A. Micheli, and P. Zoller, Nat. Phys. 3, 726 (2007).

[13] M. Wall and L. Carr, New J. Phys. 11, 055027 (2009).

[14] T. Watanabe, Phys. Rev. A 80, 053621 (2009).

[15] H. Yu, W. Liu, and C. Lee, arXiv:0910.4922 .

[16] M. L. Wall and L. D. Carr, Phys. Rev. A 82, 013611 (2010).

[17] C. Trefzger et al., New J. Phys. 12, 093008 (2010).

[18] J. Pérez-Ríos, F. Herrera, and R. Krems, New J. Phys. 12, 103007 (2010).

[19] J. Schachenmayer, I. Lesanovsky, A. Micheli, and A. Daley,
New J. Phys. 12, 103044 (2010).

[20] J. P. Kestner, B. Wang, J. D. Sau, and S. Das Sarma, Phys. Rev. B 83, 174409 (2011).

[21] A. V. Gorshkov et al., Phys. Rev. Lett. 107, 115301 (2011).

[22] A. V. Gorshkov et al., Phys. Rev. A 84, 033619 (2011).

[23] B. Yan et al., Nature 501, 521 (2013).

[24] K. R. A. Hazzard, S. R. Manmana, M. Foss-Feig, and A. M. Rey, Phys. Rev. Lett. 110, 075301 (2013).

[25] D. A. Huse et al., Phys. Rev. B 88, 014206 (2013).

[26] O. Tieleman, C. Sokos, and A. Lazarides, arXiv:1306.2810 .

[27] P. W. Anderson, Phys. Rev. 109, 1492 (1958).

[28] More generally, transitions to $m_{\ell}= \pm 1$ can be suppressed by making these off-resonant through MW dressing of the $m_{\ell}=0$ level[21].

[29] V. L. Berezinskii, Sov. Phys. JETP 32, 493 (1971).

[30] J. M. Kosterlitz and D. J. Thouless, J. Phys. C 6, 1181 (1973).

[31] C. A. Doty and D. S. Fisher, Phys. Rev. B 45, 2167 (1992).

[32] D. S. Fisher, Phys. Rev. B 50, 3799 (1994).

[33] M. V. Feigel'man, L. B. Ioffe, and M. Mézard, Phys. Rev. B 82, 184534 (2010).

[34] L. B. Ioffe and M. Mézard, Phys. Rev. Lett. 105, 037001 (2010).

[35] In the thermodynamic calculation, we treat the individual spins as classical (the limit of large $S$ ). The results for quantum spins $\left(S=\frac{1}{2}\right)$ are nearly identical. 